\title{
Alkali-ion concentration dependence of the structure of proton-conducting alkali thio-hydroxogermanates investigated with neutron diffraction
}

\author{
Maths Karlsson ${ }^{\mathrm{a}}$, Jonas Nordström ${ }^{\mathrm{a}}$, Aleksandar Matic ${ }^{\mathrm{a}}$, Steve W. Martin ${ }^{\mathrm{b}}$, Silvia Imbertic ${ }^{\mathrm{c}}$, Daniel T. Bowron ${ }^{\mathrm{c}}$ \\ ${ }^{a}$ Department of Applied Physics, Chalmers University of Technology, SE-412 96 Göteborg, Sweden \\ ${ }^{b}$ Department of Materials Science and Engineering, 2220 Hoover Hall, Iowa State University of Science and Technology, Ames, Iowa 50011 \\ ${ }^{c}$ ISIS Facility, STFC Rutherford Appleton Laboratory, Harwell Oxford, Didcot OX11 0QX, United Kingdom
}

\begin{abstract}
The proton-conducting hydrated alkali thio-hydroxogermanates $M_{x} \mathrm{GeS}_{x}(\mathrm{OH})_{4-x} \cdot y \mathrm{H}_{2} \mathrm{O}(M=\mathrm{Na}$ and $\mathrm{K} ; x=1-4, y \approx 0.5-2)$ were investigated by means of neutron diffraction with the aim to elucidate how the structure changes as a function of alkaliion concentration, $x$, type of alkali ion, $M$, and water content, $y$. For $x=1-3$ we find that the materials are amorphous and composed of thio-hydroxogermanate anions, water molecules, and charge balancing alkali ions, whereas for $x=4$ we find that the materials contain also a crystalline phase, suggesting it is difficult to prepare purely amorphous materials for the highest alkali-ion concentration, for both the $\mathrm{Na}$ and $\mathrm{K}$ based materials. For $x=1-3$, the structure is reflected by an intermediate-range ordering, with a characteristic length-scale ranging from approximately 6 to $9 \AA$, which is dependent on both $x$ and $M$ and which may be related to the separation distance between dimers of thio-hydroxogermanate anions. As $x$ increases, the intermediate-range ordering becomes more short range, possibly as the result of an increasing level of hydration water that may act as a dielectric medium that reduces the repulsive interaction between the negatively charged thio-hydroxogermanate anions and/or between the positively charged alkali ions. A comparison of the structural results to the reported conductivities of the same materials indicates a non-trivial relationship, which depends on both the type and concentration of alkali ions, as well as on the level of hydration water.
\end{abstract}

Keywords: proton conducting, thio-hydroxogermanate, neutron diffraction, fuel cell

\section{Introduction}

Research on materials applicable in clean energy technologies is one of the fastest growing areas in materials science. A particularly up-and-coming, yet challenging, future clean energy technology is the hydrogen based intermediate temperature $\left(\approx 100-300{ }^{\circ} \mathrm{C}\right)$ fuel cell. At the heart of this device is the transport of protons through a solid-state or semisolid proton-conducting electrolyte material. A promising, yet relatively unexplored, class of proton-conducting materials is hydrated alkali thio-hydroxogermanates, of the form $M_{x} \mathrm{GeS}_{x}(\mathrm{OH})_{4-x} \cdot y \mathrm{H}_{2} \mathrm{O}$, where $M=\mathrm{Na}, \mathrm{K}, \mathrm{Rb}$ or $\mathrm{Cs}, 1 \leqslant x$ $\leqslant 4$, and $y$ varies between 1 and 8.[1,2] Their conductivities typically reach a maximum value of around $10^{-3} \mathrm{Scm}^{-1}$ in the temperature range of interest $\left(\approx 100-300{ }^{\circ} \mathrm{C}\right)$ and the conductivity is related mainly to the proton bearing species and depends on both the water content and temperature.[1, 2] Recent nuclear magnetic resonance studies of the $\mathrm{Na}_{x} \mathrm{GeS}_{2}(\mathrm{OH})_{4-x} \cdot y \mathrm{H}_{2} \mathrm{O}$ ( $x=2,3$ and $y \approx 1-4)$ series suggest a proton conduction mechanism described by a combination of proton and $\mathrm{H}_{2} \mathrm{O}$ diffusion, with $\mathrm{H}_{2} \mathrm{O}$ motion limiting proton dynamics at elevated temperatures.[3] The water molecules can be removed reversibly from the materials by heating them to about $180^{\circ} \mathrm{C}$.[4] Studies have shown that the larger the value of $y$, the lower the

Email address: maths.karlsson@chalmers.se (Maths Karlsson) temperature at which the loss of water begins. [5, 4] The conductivity of the dehydrated equivalents is up to five orders of magnitude lower than for the corresponding hydrated materials, because in the dehydrated materials the alkali ions are the predominating charge carriers. $[1,2]$

Structural studies of hydrated and dehydrated alkali thiohydroxogermanates have so far focused on the $\mathrm{M}_{2} \mathrm{Ge}$ $\mathrm{S}_{2}(\mathrm{OH})_{2} \cdot y \mathrm{H}_{2} \mathrm{O}(M=\mathrm{K}, \mathrm{Rb}$ and $\mathrm{Cs})$ series, using a combination of neutron diffraction, infrared spectroscopy and firstprinciples calculations. The results from these studies suggest that the materials are amorphous and built up of dimers of thio-hydroxogermanate anions, with water molecules acting as bridges between such dimers and with the alkali ions located in "voids" formed by the network of thio-hydroxogermanate anion dimers.[6, 7] The structure of the dehydrated equivalents is build up of a percolating network of dimers of thiohydroxogermanate anions, which are linked to each other via hydrogen bonds and where the alkali ions, as for the hydrated materials, are located in "voids" formed by the thiohydroxogermanate anion dimers.[7] However, little is known about how the structure changes as a function of alkali-ion concentration. Such information is important for the understanding and further development of these materials, for example in relation to higher proton conductivity.

In this study, we therefore investigate the structure of the proton-conducting alkali thio-hydroxogermanates $M_{x} \mathrm{GeS}_{x}(\mathrm{OH})_{4-x} \cdot y \mathrm{H}_{2} \mathrm{O}$ with $M=\mathrm{Na}$ and $\mathrm{K}$ and $x=1-4$, 
Figure 1: Heating curves for (a) $\mathrm{Na}_{x} \mathrm{GeS}_{x}(\mathrm{OH})_{4-x} \cdot y \mathrm{H}_{2} \mathrm{O}(x=1-4)$ and (b) $\mathrm{K}_{x} \mathrm{GeS}_{x}(\mathrm{OH})_{4-x} \cdot y \mathrm{H}_{2} \mathrm{O}(x=1-4)$, as measured with a heating rate of $3{ }^{\circ} \mathrm{C} / \mathrm{min}$.

whereby the role of the type and concentration of alkali ion can be investigated systematically. The investigations were performed by the use of thermal analysis together with neutron diffraction. To decrease the featureless background in the neutron diffractograms, related to incoherent scattering on protons, the neutron diffraction measurements were performed on deuterated equivalents.

\section{Experimental}

\subsection{Sample preparation and thermal analysis}

The sample preparation followed the scheme by Poling et al.[1] The two $\mathrm{Na}_{x} \mathrm{GeS}_{x}(\mathrm{OD})_{4-x} \cdot y \mathrm{D}_{2} \mathrm{O}(x=1-4)$ and $\mathrm{K}_{x} \mathrm{GeS}_{x}(\mathrm{OD})_{4-x} \cdot y \mathrm{D}_{2} \mathrm{O}(x=1-4)$ series were prepared by mixing aqueous solutions (of $99.9 \% \mathrm{D}_{2} \mathrm{O}$ ) containing stoichiometric amounts of the alkali deuterosulfide $M S D$ and quartz-type $\mathrm{GeO}_{2}$ (99.998\%), whereafter the excess heavy water was allowed to evaporate at $\approx 70{ }^{\circ} \mathrm{C}$ for a period over five days. Most starting materials were purchased from Sigma-Aldrich, however, $\mathrm{KSD} \cdot \mathrm{H}_{2} \mathrm{O}$ was prepared in our own laboratory. The preparation of $\mathrm{KSD} \cdot \mathrm{H}_{2} \mathrm{O}$ was done by first dissolving $\mathrm{KOH}(\geqslant 90 \%$, Sigma-Aldrich) in methanol, followed by bubbling of $\mathrm{H}_{2} \mathrm{~S}$ gas through the solution for a period over $3 \mathrm{~h}$. The methanol was then allowed to evaporate from this solution overnight by a gentle stream of $\mathrm{N}_{2}$ gas above the solution. Subsequently, KSD was prepared by dissolving the non-deuterated equivalent in $\mathrm{D}_{2} \mathrm{O}$, followed by gentle rotary evaporation of the molecular water. The latter procedure was repeated three times in order to allow all $\mathrm{H}$ to be exchanged with $\mathrm{D}$. The corresponding reaction for the synthesis of the deuterated alkali thio-hydroxogermanates may be written as

$$
\begin{gathered}
x M S D+\mathrm{GeO}_{2}+y \mathrm{D}_{2} \mathrm{O} \rightarrow \mathrm{M}_{x} \mathrm{GeS} \mathrm{S}_{x}(\mathrm{OD})_{4-x} \cdot y \mathrm{D}_{2} \mathrm{O} \\
x=1,2,3,4 ; \mathrm{M}=\mathrm{Na}, \mathrm{K},
\end{gathered}
$$

where the number of $\mathrm{D}_{2} \mathrm{O}$ molecules $(y)$ was determined by means of thermal gravimetric analysis (TGA), using the TG 209 F1 Iris instrument from Netzsch. For each TGA measurement, about $20 \mathrm{mg}$ of sample was placed in an open aluminum pan, which was heated under a flow of He gas $(25 \mathrm{ml} / \mathrm{min})$ from room temperature up to $70{ }^{\circ} \mathrm{C}$. At this temperature, the samples were kept for $60 \mathrm{~min}$, followed by further heating up to $500{ }^{\circ} \mathrm{C}$, with a heating rate of $3{ }^{\circ} \mathrm{C} / \mathrm{min}$. The heating curves for all the samples that were investigated are shown in Figure 1 The $y$ values were estimated from the mass loss during the temperature increase from 70 to $500{ }^{\circ} \mathrm{C}$. Table 1 displays the $y$ values obtained for all samples and also displays data for the bulk and atomic densities. The densities were determined using a gas-pycnometer "AccuPyc II 1340 Pycnometer" from Micrometrics.

\subsection{Neutron diffraction}

The neutron diffraction experiment was performed at the SANDALS diffractometer of the ISIS neutron facility of Rutherford Appleton Laboratory, UK. The SANDALS diffractometer, described in detail in Ref. [8], receives neutrons with wavelengths from 0.05 to $5 \AA$. The 660 detectors are summed into 18 groups, which cover an angular range of 3.8-39 , yielding scattering vectors $(Q)$ in the range $0.1-50 \AA^{-1}$. The samples were ground into fine powders using an agate mortar and a pestle, and loaded in flat sample cells of a $68 \%$ titanium and $32 \%$ zirconium alloy, which does not contribute to any coherent scattering. The sample thickness was between 2 and $4 \mathrm{~mm}$, with a typical measuring time between 6 and $12 \mathrm{~h}$, and all measurements were performed at room temperature. Measurements of the empty sample cans, the empty sample chamber, and on a vanadium standard, were used for correcting for the background and detector efficiency, and hence for normalization of the data onto an absolute scale of barns per steradian per atom. Using the Gudrun software,[9] as developed for the SANDALS diffractometer, the raw data was corrected for multiple scattering, absorption, inelasticity effects, that generates a fully corrected structure factor, $S(Q)$, suitable for analysis in terms of the atomic pair-correlations. The level of the measured differential neutron scattering cross-section, related to the $S(Q)$, was well within the range of expected values, which shows that the data reduction and hence the sample composition and density were correct. The corresponding pair-correlation functions, $G(r)$, were obtained by Fourier transformation according to the following:

$$
G(r)=1+\frac{\sum_{i=1}^{n} c_{i} \bar{b}_{i}^{2}}{\left(\sum_{i=1}^{n} c_{i} \bar{b}_{i}\right)^{2}} \frac{1}{2 \pi^{2} \rho_{0} r} \int_{0}^{\infty}[S(Q)-1] \sin (Q r) Q d Q,
$$

where $c_{i}$ and $b_{i}$ denote the concentration and scattering length of each component $i$, respectively, in a multicomponent system of scatterers, and $\rho_{0}$ denotes the average atomic number density.

\section{Results}

\subsection{Structure factors}

In Figure 2, we present the total neutron structure factors, $S(Q)$ s, of the two investigated alkali thio-hydroxogermanate systems for the four different values of alkali-ion concentration $(x=1-4)$. As can be seen in the figure, the oscillations in $S(Q)$ persist up to a $Q$-value of $20 \AA^{-1}$, suggesting a well defined short-range structure for all the investigated materials. The smooth oscillations for the $x=1-3$ materials are in agreement with an amorphous structure, as observed in previous investigations using X-ray diffraction $(x=1-3)$ and neutron diffraction $(x=2)$ techniques.[1, 7] However, for $x=4$ we observe for both the $\mathrm{Na}$ and $\mathrm{K}$ based materials that the structure factor contains a number of Bragg peaks for $Q \lesssim 5 \AA^{-1}$, superimposed on a smoothly varying diffraction pattern. The presence of Bragg peaks reveals a crystalline phase in addition 
Figure 2: Structure factors of $\mathrm{Na}_{x} \mathrm{GeS}_{x}(\mathrm{OD})_{4-x} \cdot y \mathrm{D}_{2} \mathrm{O}(x=1-4)$ and $\mathrm{K}_{x} \mathrm{GeS}_{x}(\mathrm{OD})_{4-x} \cdot y \mathrm{D}_{2} \mathrm{O}(x=1-4)$. For clarity, the structure factors have been separated vertically by 0.5 .

Figure 3: Structure factors (a) and pair-correlation functions (b) of $\mathrm{Na}_{x} \mathrm{GeS}_{x}(\mathrm{OD})_{4-x} \cdot y \mathrm{D}_{2} \mathrm{O}(x=1-3)$ and $\mathrm{K}_{x} \mathrm{GeS}_{x}(\mathrm{OH})_{4-x} \cdot y \mathrm{H}_{2} \mathrm{O}(x=1-3)$. For clarity, the structure factors and pair-correlation functions have been separated vertically by 0.5 and 2.0 , respectively. Vertical lines serve as guidance.

to an amorphous phase relating to a disordered network of alkali thio-hydroxogermanate anions. Due to the limited $Q$ resolution of the SANDALS diffractometer to resolve Bragg peaks, we make no attempt to here further analyze the structures of these materials, but rather conclude that it is difficult to prepare purely amorphous materials for the highest alkali-ion concentration, for both types of alkali ion as here investigated. In the following we therefore focus on the two $x=1-3$ series, i.e. $\mathrm{Na}_{x} \mathrm{GeS}_{x}(\mathrm{OD})_{4-x} \cdot y \mathrm{D}_{2} \mathrm{O}(x=1-3)$ and $\mathrm{K}_{x} \mathrm{GeS}_{x}(\mathrm{OD})_{4-x} \cdot y \mathrm{D}_{2} \mathrm{O}$ $(x=1-3)$.

Regarding the high- $Q$ range, $>10 \AA^{-1}$, we observe small, but significant, differences in the structure factor amongst the different materials. In particular, we observe that the oscillations at high $Q$ seem to be more pronounced for the lower $x$ values, indicating that the short-range structure is more well defined for these materials. Figure 3(a) shows a close up of the low- $Q$ range of the $S(Q)$ s, for both systems. The major peaks in the $S(Q)$ s are located at approximately $0.7,2.0,4.5$ and at $7.7 \AA^{-1}$, respectively, with the two latter peaks being relatively broad. The intensity and positions of all four peaks are found to vary significantly both with the type and concentration of alkali ion (Na, K, $x=1-3$ ).

Considering the $\mathrm{Na}$ based system, we observe that the first sharp diffraction peak (FSDP) is located at $0.73 \AA^{-1}$ for $x=1$ and decreases in intensity and shifts to a $Q$-value of $0.95 \AA^{-1}$ for $x=3$. The peak positions for the three major peaks are for both systems reported in Table 2 . As can be seen, the second peak in $S(Q)$ shifts from $1.99 \AA^{-1}$ for $x=1$ to $2.15 \AA^{-1}$ for $x=3$, whereas its intensity remains essentially unchanged. Regarding the two weaker, broader, peaks at 4.5 and $7.7 \AA^{-1}$, these are found to decrease in intensity with $x$, as well as they split into two weaker components for $x=2$ and $x=3$. Considering the $\mathrm{K}$ based system, the FSDP shifts from $0.80 \AA^{-1}$ at $x=1$ to $1.13 \AA^{-1}$ at $x=3$, and the second peak shifts from $2.03 \AA^{-1}$ to $2.18 \AA^{-1}$, cf. Table 2, which is similar to the $\mathrm{Na}$ based system. Most noticeable is the difference in intensity of all peaks, which are found to be lower for the Na based system compared to the $\mathrm{K}$ based equivalent. Considering the fact that the coherent scattering lengths for $\mathrm{Na}$ (1.66 barns) and $\mathrm{K}$ (1.69 barns) are similar, this indicates a higher degree of disorder in the $\mathrm{Na}$ based system.

\subsection{Pair-correlation functions}

The total pair-correlation functions of the $\mathrm{Na}_{x} \mathrm{GeS}_{x}(\mathrm{OD})_{4-x} \cdot y \mathrm{D}_{2} \mathrm{O}(x=1-3)$ and $\mathrm{K}_{x} \mathrm{GeS}_{x}(\mathrm{OH})_{4-x} \cdot y \mathrm{H}_{2} \mathrm{O}$ $(x=1-3)$ series are shown in Figure 3(b). The pair-correlation functions are for all the investigated materials characterized by two strong peaks at $\approx 1 \AA$ and $\approx 1.8 \AA$, respectively, and a broader, weaker, peak at $\approx 3.3 \AA$. On the basis of the previous report on $\mathrm{K}_{2} \mathrm{GeS}_{2}(\mathrm{OD})_{2} \cdot y \mathrm{D}_{2} \mathrm{O}$, the peak at $\approx 1 \AA$ is assigned to $\mathrm{O}-\mathrm{D}$ correlations, the peak at $\approx 1.5 \AA$ is assigned to D-D correlations of water molecules, the peak at $\approx 1.8 \AA$ is assigned to Ge-O correlations, the peak at $\approx 2.2 \AA$ is assigned to $\mathrm{Ge}-\mathrm{S}$ correlations, and the broad $3.3 \AA$ peak is assigned to O-S correlations and different correlations involving the alkali ions.[7] Following further the assignment in our previous report on $\mathrm{K}_{2} \mathrm{GeS}_{2}(\mathrm{OD})_{2} \cdot y \mathrm{D}_{2} \mathrm{O}$,[7] the two shoulders of the broad peak centered at $\approx 3.3 \AA$ can be assigned to $\mathrm{Na} / \mathrm{K}-\mathrm{O}$ correlations $(r \approx 2.8 \AA)$ and $\mathrm{Na} / \mathrm{K}-\mathrm{S}$ correlations $(r \gtrsim 3.2 \AA)$, respectively. Depending on the coordination number and type of alkali ion, these correlations are expected at slightly different distances in the respective pair-correlation functions. Specifically, by using the ionic radii reported by Shannon,[10] $\mathrm{Na}-\mathrm{O}$ and K-O correlations are expected at 2.3-2.7 $\AA$ and at 2.7-3.0 $\AA$, respectively, which indeed agree well with the shoulder on the low $-r$ side of the main peak at $\approx 3.3 \AA$. Similarly, Na-S and K-S correlations are expected at 2.8-3.2 $\AA$ and at 3.2-3.5 $\AA$, respectively, which agree well with the shoulder on the high- $r$ side of the same peak.

Upon increasing the concentration of the alkali ion, the major change of the $G(r)$ s for both systems relate to changes in the intensity of the pair-correlation peaks. In particular, we observe a large increase in intensity of the O-D correlation peak at $\approx 1 \AA$, as well as a simultaneous decrease in intensity of the $\mathrm{Ge}-\mathrm{O}$ correlation peak at $\approx 1.8 \AA$. For both systems, we also observe how the intensity of the Ge-S correlation peak at $\approx 2.2 \AA$ increases from being practically non-observable for $x=1$ to become almost as strong as the Ge-O correlation peak at $x=3$, and that the intensity of the $\mathrm{Na} / \mathrm{K}-\mathrm{O}$ correlation peak at around $2.8 \AA$ decreases in intensity with increasing $x$ to vanish completely at $x=3$. The gradual intensity increase of the O-D correlation peak with increasing $x$ is consistent with the increasing number of $\mathrm{D}_{2} \mathrm{O}$ molecules as $x$ increases. The decreasing $\mathrm{Ge}-\mathrm{O}$ correlation peak and increasing $\mathrm{Ge}-\mathrm{S}$ correlation peak is consistent with the increasing substitution of -OD groups with -S groups on the thio-hydroxogermanate anions. Similarly, the $\mathrm{Na} / \mathrm{K}-\mathrm{O}$ correlation peak becomes suppressed with increasing $x$ as a consequence of the decreasing number of $\mathrm{O}$ on the thiohydroxogermanate anions. The latter idea is supported by the fact that the positively charged alkali ions most likely tend to coordinate to the negatively charged sulfide anions on the thiohydroxogermanate anions. Furthermore, we observe a slight shift of the Ge-O correlation peak when $x$ increases, see Table 2. Finally, we observe that the intensity of the O-D correlation peak is systematically more intense for the $\mathrm{K}$ based system compared to the Na-based one, even though the $\mathrm{D}_{2} \mathrm{O}$ content (y) is almost the same. The could relate to a slightly different coordination of the D-O pairs in the two systems. This is in agreement with the structure factors in Figure 3(a), showing 
that the peaks for the $\mathrm{Na}$ based system are generally less intense. On the other hand, it could also be a consequence of the presence of some hydrogen rather than only deuterium in the Na based system, since the negative scattering length of hydrogen would reduce the height of the O-D peak in the system. It should be noted, however, that the scattering level determined in the Gudrun analysis is in agreement with fully deuterated samples, although some trace amounts of hydrogen can be expected.

\section{Discussion}

By combining the results obtained from the thermal analysis (Figure 1) and neutron scattering experiments (Figure 2 and Figure 3) we are now able to reveal several important aspects related to the alkali-ion concentration dependence of the structure of proton-conducting alkali thio-hydroxogermanates. A direct interpretation of the diffraction peaks of the total structure factors into real space is, however, in general difficult since this conversion involves a Fourier transformation. Nevertheless, the position of the FSDP, located at $Q_{1}$, can for amorphous materials be related to an intermediate-range ordering in real space, with a characteristic length-scale of about $2 \pi / Q_{1} \cdot[11]$ In this context, the previous neutron diffraction and modeling study of $\mathrm{Cs}_{2} \mathrm{GeS}_{2}(\mathrm{OH})_{2} \cdot y \mathrm{H}_{2} \mathrm{O}$, suggests that the intermediate-range ordering is associated with the distance between dimers of thio-hydroxogermanate anions as well as to Ge-Ge correlations.[6, 7] For the $\mathrm{Na}$ based series, this corresponds to a length-scale of about $8.6 \AA$ at $x=1$ and $6.6 \AA$ at $x=3$, whereas for the $\mathrm{K}$ based series for which a similar effect of the FSDP is observed, the intermediate-range ordering changes from about $7.9 \AA$ at $x=1$ to $5.6 \AA$ at $x=3$. The intermediate-range ordering is hence somewhat more longrange for the $\mathrm{Na}$ based series compared to the $\mathrm{K}$ based equivalent, and contracts as $x$ increases for both series of materials. Since the ionic radius of $\mathrm{K}$ is considerable larger than that of $\mathrm{Na},[10]$ this is an important result demonstrating that the radius of the alkali-ion is not a parameter that determines the lengthscale of the intermediate-range ordering. Instead, we note that the fact that an increase of $x$ is accompanied by an increase of the level of hydration water [cf. Table 1], may be indicative of the fact that the water molecules may act as a dielectric medium that decreases the repulsive interaction between the negatively charged thio-hydroxogermanate anions and/or between the positively charged alkali ions. This is in agreement with the previous neutron diffraction study on $M_{2} \mathrm{GeS}_{2}(\mathrm{OH})_{2} \cdot y \mathrm{H}_{2} \mathrm{O}(M=\mathrm{K}$, $\mathrm{Rb}$ and $\mathrm{Cs}$ ), for which the respective FSDPs were found to shift to lower $Q$-values upon dehydration of the materials.[7] In this context, one should note that the position and shape of the peaks in $S(Q)$ for a multicomponent system have contributions from a number of partial structure factors and in our case the weighting factors of the respective partial pair-correlation functions are shown in Table 3. Therefore, the difference in positions of the diffraction peaks may be (partially) related to the difference in intensity of the contributing pair-correlation functions, even though the underlaying structure remains basically the same. In our case, we observe especially that the contributions from pair-correlations involving deuterium, in particular O-D and D$\mathrm{D}$, are for all materials dominating the scattering, however they do become less pronounced with increasing $x$. Instead, the sulfur and alkali-ion containing pair-correlation functions increase in strength as $x$ increases and in effect the weighting factors overall become more similar to each other. This is in agreement with the overall less intense and less distinct peaks in the structure factor as $x$ increases, suggesting that the intermediaterange ordering becomes gradually less defined. This may be attributed to small structural rearrangements of the percolating network built up of thio-hydroxogermanate anions and water molecules.

A comparison of our structural results with the reported conductivities of the investigated materials provides some insight into the nature of proton transport in these materials. Literature data of the proton conductivity as a function of temperature exists for $\mathrm{Na}_{x} \mathrm{GeS}_{x}(\mathrm{OH})_{4-x} \cdot y \mathrm{H}_{2} \mathrm{O}(x=$ 2,3) and $\mathrm{K}_{x} \mathrm{GeS}_{x}(\mathrm{OH})_{4-x} \cdot y \mathrm{H}_{2} \mathrm{O}(x=2,3)$.[1] In Table 4 are shown the conductivities as measured at a temperature of $100{ }^{\circ} \mathrm{C}$. Included in Table 4 are also a comparison of the deuterium (hydrogen) concentration as determined in our work and in ref.[1] Interestingly, the conductivities are essentially the same for $\mathrm{Na}_{x} \mathrm{GeS}_{x}(\mathrm{OH})_{4-x} \cdot y \mathrm{H}_{2} \mathrm{O}(x=2,3)$ and $\mathrm{K}_{3} \mathrm{GeS}_{3}(\mathrm{OH}) \cdot y \mathrm{H}_{2} \mathrm{O}$, despite the relatively large variation in the level of hydration water amongst the different materials, whereas for $\mathrm{K}_{2} \mathrm{GeS}_{2}(\mathrm{OH})_{2} \cdot y \mathrm{H}_{2} \mathrm{O}$ the conductivity is about three orders of magnitude lower. In this context, it has been suggested recently that the proton conduction is governed by a combination of proton and $\mathrm{H}_{2} \mathrm{O}$ diffusion, with $\mathrm{H}_{2} \mathrm{O}$ motion limiting proton dynamics at elevated temperatures, [3] therefore the presence of hydration water is essential for high proton conductivity. The similar proton conductivities for three of the materials as investigated here $\left(\mathrm{Na}_{x} \mathrm{GeS}_{x}(\mathrm{OH})_{4-x} \cdot y \mathrm{H}_{2} \mathrm{O}(x=2,3)\right.$ and $\left.\mathrm{K}_{3} \mathrm{GeS}_{3}(\mathrm{OH}) \cdot y \mathrm{H}_{2} \mathrm{O}\right)$ may therefore suggest that there is a threshold water concentration, above which the level of proton conductivity is high and largely independent of $y$. According to Table 4, such a threshold concentration may lay in the range $y=0.8-1.3$. However, this is in disagreement with the $\mathrm{Cs}_{2} \mathrm{GeS}_{2}(\mathrm{OH})_{2} \cdot y \mathrm{H}_{2} \mathrm{O}$ material, which shows the highest conductivity and lowest water concentration $(y=0.2-0.6)$ of all materials. This would indicate that the threshold water concentration depends on both the type and concentration of alkali ion, but evidently, the level of hydration water is certainly not the only factor of play. In fact, our results suggest a complicated relationship between structure and conductivity. Further investigations of the mechanistic aspects of proton dynamics, using e.g. quasielastic neutron scattering together with molecular dynamics simulations, [12] are hence likely to be rewarding.

\section{Conclusions}

The new ceramic proton-conducting hydrated alkali thiohydroxogermanates $M_{x} \mathrm{GeS}_{x}(\mathrm{OH})_{4-x} \cdot y \mathrm{H}_{2} \mathrm{O}(M=\mathrm{Na}$ and $\mathrm{K}$; $x=1-4, y \approx 0.5-2$ ) have been investigated by means of thermal analysis and neutron diffraction with the aim to elucidate how the structure changes as a function of alkali-ion concentration, $x$, type of alkali ion, $M$, and water content, $y$. For 
$x=1-3$, the results show that the materials are amorphous and composed of thio-hydroxogermanate anions, water molecules, and charge balancing alkali ions, whereas for $x=4$ we find that the materials contain also a crystalline phase, suggesting it is difficult to prepare purely amorphous materials for the highest alkali-ion concentration, for both the $\mathrm{Na}$ and $\mathrm{K}$ based materials. For $x=1-3$, the structure is reflected by an intermediaterange ordering, with a characteristic length-scale ranging from approximately 6 to $9 \AA$, which is dependent on both $x$ and $M$ and which may be related to the separation distance between dimers of thio-hydroxogermanate anions. As $x$ increases, the intermediate-range ordering shortens, most likely as the result of an increasing level of hydration water that is suggested to act as a dielectric medium that reduces the repulsive interaction between the negatively charged thio-hydroxogermanate anions and/or between the positively charged alkali ions. A comparison of the structural results to the reported conductivities of the same materials indicates a non-trivial relationship between structure and conductivity, which depends on both the type and concentration of alkali ion, as well as the presence of molecular water in the material.

\section{Acknowledgments}

This work was primarily supported by the Swedish Research Council (grant nr. 2010-3519 and 2011-4887) and the United States Department of Energy's Hydrogen Program under Cooperative Agreement No. DE-FC36-00GO1-531. The STFC Rutherford Appleton Laboratory is thanked for access to neutron beam facilities (RB 1010034).

[1] S. Poling, C. Nelson, and S. W. Martin, Chem. Mater. 17, 1728 (2005).

[2] S. A. Poling, C. R. Nelson, and S. W. Martin, Mater. Lett. 60, 23 (2006).

[3] A. Shastri, D. Shane, J. Nordström, A. Matic, and S. W. Martin, Solide State Ion. 228, 46 (2012).

[4] M. Karlsson, C. R. Nelson, C. A. Martindale, S. W. Martin, A. Matic, and L. Börjesson, Solid State Ion. 177, 1009 (2006).

[5] C. R. Nelson, S. Olson, S. A. Poling, and S. W. Martin, Chem. Mater. 18, 6436 (2006).

[6] M. Karlsson, A. Matic, C. R. Nelson, C. A. Martindale, D. T. Bowron, S. W. Martin, and L. Börjesson, Solid State Ion. 178, 501 (2007).

[7] M. Karlsson, A. Matic, I. Panas, D. T. Bowron, S. W. Martin, C. R. Nelson, C. A. Martindale, A. Hall, and L. Börjesson, Chem. Mater. 20, 6014 (2008).

[8] C. Benmore and A. Soper, The SANDALS Manual (ISIS Facility, Rutherford Appleton Laboratory, 1998).

[9] Soper, A. Gudrun is a suite of programs with a graphic interface for the full correction of neutron diffraction data from various instruments at ISIS pulsed spallation neutron source; http://www.isis.stfc.ac.uk/groups/disordered-materials/.

[10] R. D. Shannon, Acta Cryst. A 32, 751 (1976).

[11] S. R. Elliot, Physical Review Letters 67, 711 (1991).

[12] M. Karlsson, Phys. Chem. Chem. Phys. 17, 26 (2015). 
Table 1: Densities and deuterium content of deuterated alkali thiohydroxogermanates.

\begin{tabular}{lccc}
\hline \hline Material & $\begin{array}{c}\text { Bulk density } \\
\left(\mathrm{g} / \mathrm{cm}^{3}\right)\end{array}$ & $\begin{array}{c}\text { Atomic no. } \\
\text { density }\left(\text { atoms } / \AA^{3}\right)\end{array}$ & $y$ \\
\hline $\mathrm{NaGeS}(\mathrm{OD})_{3} \cdot y \mathrm{D}_{2} \mathrm{O}$ & 2.801 & 0.0988 & 0.6 \\
$\mathrm{Na}_{2} \mathrm{GeS}_{2}(\mathrm{OD})_{2} \cdot y \mathrm{D}_{2} \mathrm{O}$ & 2.486 & 0.0788 & 1.3 \\
$\mathrm{Na}_{3} \mathrm{GeS}_{3}(\mathrm{OD}) \cdot y \mathrm{D}_{2} \mathrm{O}$ & 2.311 & 0.0686 & 1.9 \\
$\mathrm{Na}_{4} \mathrm{GeS}_{4} \cdot y \mathrm{D}_{2} \mathrm{O}$ & 2.285 & 0.0585 & 1.8 \\
\hline $\mathrm{KGeS}(\mathrm{OD})_{3} \cdot y \mathrm{D}_{2} \mathrm{O}$ & 2.649 & 0.0820 & 0.5 \\
$\mathrm{~K}_{2} \mathrm{GeS}_{2}(\mathrm{OD})_{2} \cdot y \mathrm{D}_{2} \mathrm{O}$ & 2.657 & 0.0736 & 0.8 \\
$\mathrm{~K}_{3} \mathrm{GeS}_{3}(\mathrm{OD}) \cdot y \mathrm{D}_{2} \mathrm{O}$ & 2.265 & 0.0551 & 1.7 \\
$\mathrm{~K}_{4} \mathrm{GeS}_{4} \cdot y \mathrm{D}_{2} \mathrm{O}$ & 2.235 & 0.0427 & 1.2 \\
\hline \hline
\end{tabular}

Table 2: Peak positions for the first three peaks in $S(Q)$ and for the first two strong peaks in $G(r)$. Note that the third diffraction peak, centered at $Q_{3}$, splits into two peaks for $x=2$ and $x=3$. The uncertainties for the positions in $S(Q)$ and $G(r)$ are \pm 0.02 and \pm 0.01 , respectively.

\begin{tabular}{lccccc}
\hline Na series & & & & & \\
$x$ & $Q_{1}\left(\AA^{-1}\right)$ & $Q_{2}\left(\AA^{-1}\right)$ & $Q_{3}\left(\AA^{-1}\right)$ & $r_{1}(\AA)$ & $r_{2}(\AA)$ \\
\hline 1 & 0.73 & 1.99 & $\approx 4.5$ & 0.96 & 1.77 \\
2 & 0.78 & 2.10 & $\approx 3.7, \approx 4.5$ & 0.95 & 1.77 \\
3 & 0.95 & 2.15 & $\approx 3.5, \approx 4.3$ & 0.95 & 1.80 \\
\hline K series & & & & & \\
$x$ & $Q_{1}\left(\AA^{-1}\right)$ & $Q_{2}\left(\AA^{-1}\right)$ & $Q_{3}\left(\AA^{-1}\right)$ & $r_{1}(\AA)$ & $r_{2}(\AA)$ \\
\hline 1 & 0.80 & 2.03 & $\approx 4.5$ & 0.96 & 1.77 \\
2 & 0.93 & 2.13 & $\approx 4.3$ & 0.96 & 1.79 \\
3 & 1.13 & 2.18 & $\approx 3.5, \approx 4.2$ & 0.96 & 1.80 \\
\hline \hline
\end{tabular}

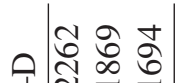

1

mªt

वे त्रे तु

000

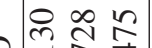

$1=0.000 .000$

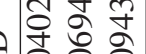

un 0 : 0 :

ॠ $m$ aे

1 O

0

ț $\bar{m}$

u 8 \&

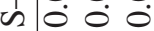

in

$8 \begin{array}{lll}1 & 0 & 0 \\ 0 & 0 & 0 \\ 0 & 0 & 0\end{array}$

츤ำ

ऽ)

\%

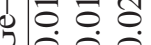

ঠ઼

0 o. 0.

000

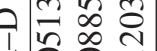

乙

ํํำ

๘

웅

๘ ठ0

00

ঠ유 윳

ठै ठิ है

ิㅗㅇำ

孚
ค|

000

○े

ชำ

이 웅

100.

호 이

१

곡ำ

व ग ग

o $\infty$

थ

00

ใฺ

ல

0 㢳

S

ข

(1)

S낭요

80.0

$\infty$

1

오온

1 垈

능 융

4 ऽ

ษ年告

4 .

$m=N$

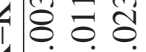

1 웅 
Table 4: Summary of deuterium content for selected compositions as determined in this work, as well as hydrogen content as reported in ref.[1], for selected compositions. Included in the table is literature data of the conductivities measured on hydrated samples at $100{ }^{\circ} \mathrm{C}$.[1]

\begin{tabular}{lccc}
\hline \hline Material & $y$ (this work) & $y$ (ref.[1]) & $\begin{array}{c}\text { Conductivity } \\
\left(\mathrm{Scm}^{-1}\right)\end{array}$ \\
\hline $\mathrm{Na}_{2} \mathrm{GeS}_{2}(\mathrm{OD})_{2} \cdot y \mathrm{D}_{2} \mathrm{O}$ & 1.3 & $\approx 1.3$ & $\approx 5 \cdot 10^{-3}$ \\
$\mathrm{~K}_{2} \mathrm{GeS}_{2}(\mathrm{OD})_{2} \cdot y \mathrm{D}_{2} \mathrm{O}$ & 0.8 & $0.4-0.8$ & $\approx 1 \cdot 10^{-6}$ \\
$\mathrm{Na}_{3} \mathrm{GeS}_{3}(\mathrm{OD}) \cdot y \mathrm{D}_{2} \mathrm{O}$ & 1.9 & $3.8-4.4$ & $\approx 1 \cdot 10^{-3}$ \\
$\mathrm{~K}_{3} \mathrm{GeS}_{3}(\mathrm{OD}) \cdot y \mathrm{D}_{2} \mathrm{O}$ & 1.7 & $2.0-2.2$ & $\approx 1 \cdot 10^{-3}$ \\
\hline \hline
\end{tabular}




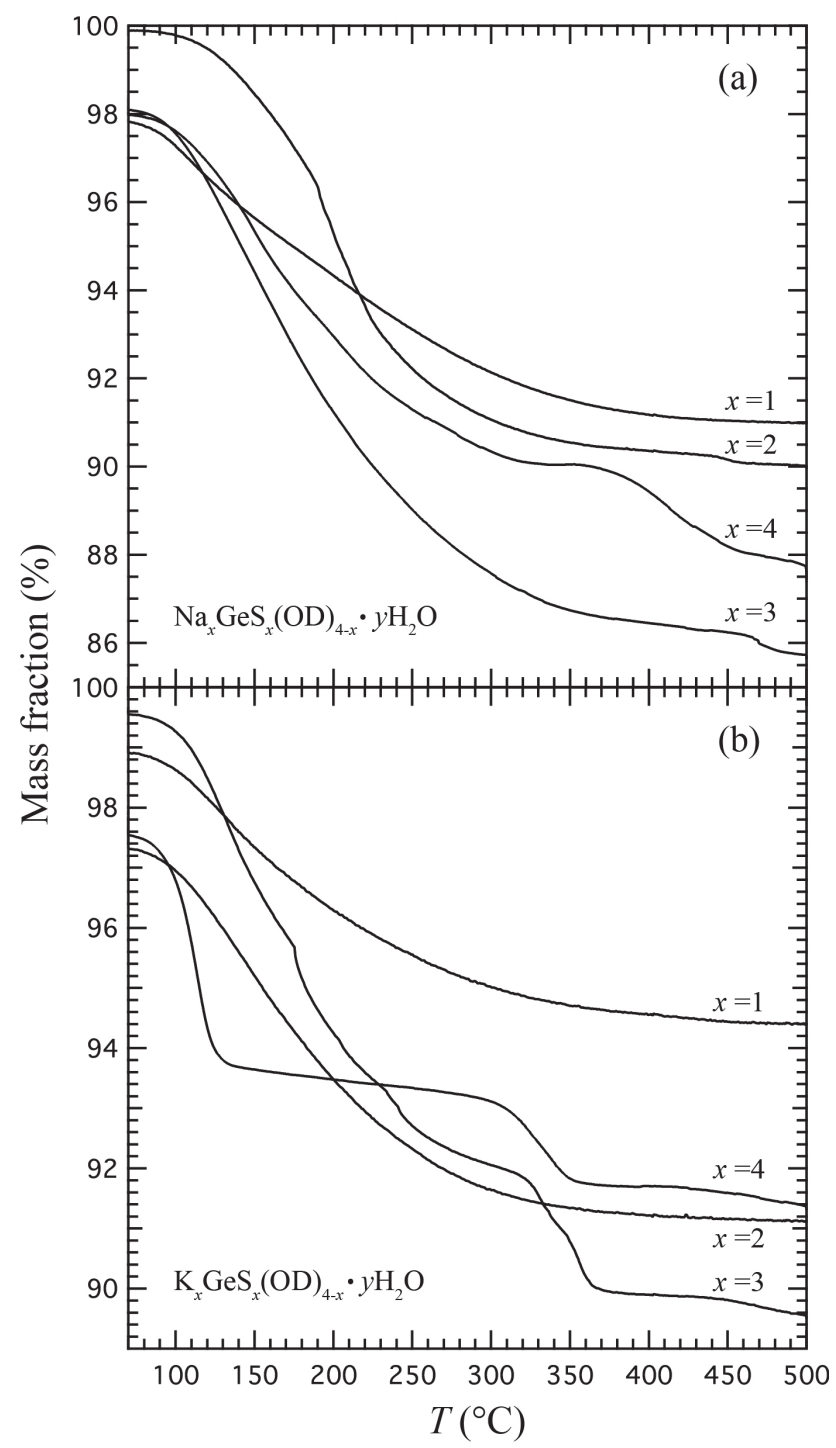

Figure 1: Heating curves for (a) $\mathrm{Na}_{x} \mathrm{GeS}_{x}(\mathrm{OH})_{4-x} \cdot y \mathrm{H}_{2} \mathrm{O}(x=1-4)$ and (b) $\mathrm{K}_{x} \mathrm{GeS}_{x}(\mathrm{OH})_{4-x} \cdot y \mathrm{H}_{2} \mathrm{O}(x=1-4)$, as measured with a heating rate of $3{ }^{\circ} \mathrm{C} / \mathrm{min}$. 


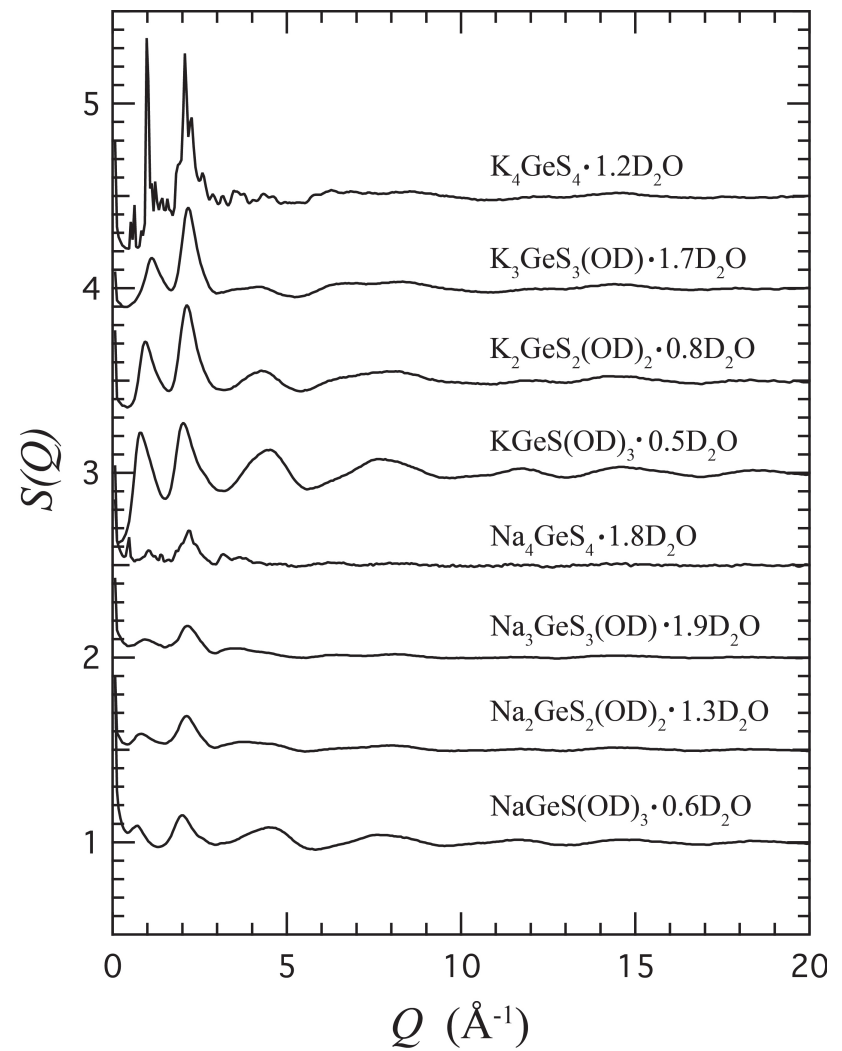

Figure 2: Structure factors of $\mathrm{Na}_{x} \mathrm{GeS}_{x}(\mathrm{OD})_{4-x} \cdot y \mathrm{D}_{2} \mathrm{O}(x=1-4)$ and $\mathrm{K}_{x} \mathrm{GeS}_{x}(\mathrm{OD})_{4-x} \cdot y \mathrm{D}_{2} \mathrm{O}(x=1-4)$. For clarity, the structure factors have been separated vertically by 0.5 . 

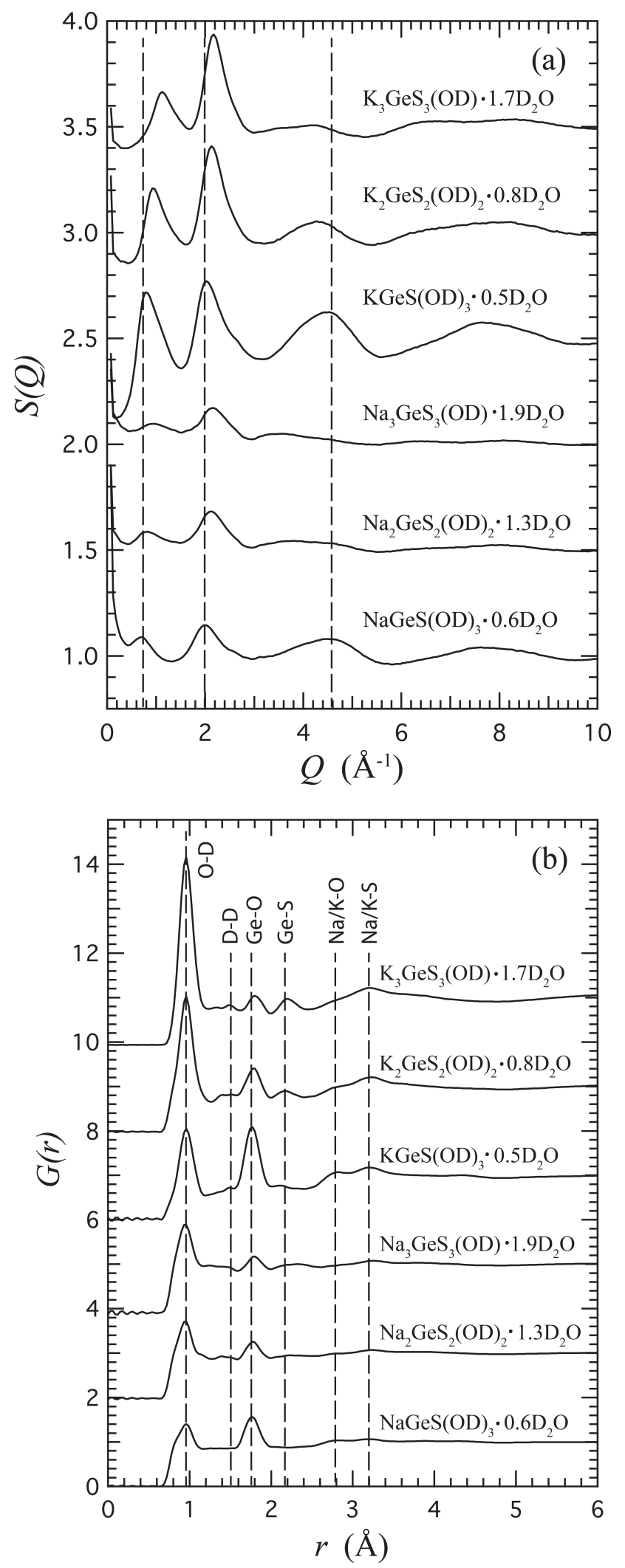

Figure 3: Structure factors (a) and pair-correlation functions (b) of $\mathrm{Na}_{x} \mathrm{GeS}_{x}(\mathrm{OD})_{4-x} \cdot y \mathrm{D}_{2} \mathrm{O}(x=1-3)$ and $\mathrm{K}_{x} \mathrm{GeS}_{x}(\mathrm{OH})_{4-x} \cdot y \mathrm{H}_{2} \mathrm{O}(x=1-3)$. For clarity, the structure factors and pair-correlation functions have been separated vertically by 0.5 and 2.0 , respectively. Vertical lines serve as guidance. 